2017-2018. Median age (range) at start of treatment was 14.8 yrs $(9.7,18.0)$ and this remained unchanged during 2011-2018 $(\mathrm{p}=0.543)$. GnRHa was discontinued in $5 / 65$ (8\%) YP [1 (2\%) birth-assigned female, 4 (19\%) birthassigned males, $\mathrm{p}=0.018$ ] after a median (range) duration of 0.8 yrs $(0.5,1.5)$. Twenty-two YP started gender-affirming hormones (17 birth-assigned females, 5 birth-assigned males). Median age (range) at start was 16.21 yrs $(16.04,17.13)$. In one YP, this was discontinued, after a period of 0.33 yrs. Overall, 3/74 (4\%) YP (1 birth-assigned female, 2 birth-assigned males) were no longer experiencing gender dysphoria during the period of attending paediatric endocrinology and discontinued treatment.

Conclusion There has been a marked increase from 2016 onward in prevalence of YP with GD referred to paediatric endocrinology, requiring an evolution in the structure of a whole service. Pursuit of fertility preservation has been poor and requires closer consideration.

\section{G432(P) A STRATEGY FOR REDUCING PLANNED STABILIZATION ADMISSIONS FROM TWO WEEKS TO ONE}

${ }^{1} V$ Dublon, ${ }^{2} S$ Green, ${ }^{1} \mathrm{M}$ Benitez-Castillo. ${ }^{1}$ Department of Child Health, Royal Free Hospital NHS Trust, London, UK; ${ }^{2}$ Royal Free Hospital School, Royal Free Hospital NHS Trust, London, UK

\subsection{6/archdischild-2020-rcpch.373}

Introduction Hospital schools can provide structure and learning for young people throughout a planned admission. As integral part of the diabetes multidisciplinary team (MDT), through careful planning and coordination of clinical visits we condensed our two week admissions in to one week, thus improving the speed and quality of the structured education for young people and their families. This has clear economical, educational and time savings for both the young person, their families and the NHS.

Method Working closely with the MDT, the hospital school were given responsibility to assist with the following:

- Co-produce an MDT timetable of appointments during the admission, prior to them being admitted

- With consent, contact their school so work can be arranged for the admission to ensure no child falls behind or is forgotten.

- Screening for Dyslexia and Dyscalculia to gauge the best way in a short time to support them with carb counting, insulin ratios and correction doses.

- Complete before and after questionnaires regarding diabetes knowledge.

- Complete 'All About Me' form so we are very much putting the young person needs central.

- Support technology, teaching how to upload to Diasend or similar.

- Share observations with the MDT.

- Advise regarding diabetes charities and Disability Living Allowance.

- Share a mini library, funded by school, of diabetes related books.

- Provide information regarding social and education/ information days.

- Complete before/after and patient experience questionnaires.

- Explain Access Arrangements and Special consideration for SATS, GCSEs and A-level examinations.
Results We have an abundance of quotes and very positive graphical/pictorial data from young people, parents and staff.

Conclusion Hospitals schools can make admissions more planned and particularly young people appreciate this. Every parent, young person and MDT, deemed the hospital school a vital resource in providing structure, information and guidance during planned admissions.

Additionally their teaching expertise helped immensely in both the teaching and assessment of learning, again allowing us to condense the time needed.

They are additional watchers, having a constant ward presence, leading to more effective overall care, which leads to shorter stays, effective education, reductions in emergency admissions and subsequent saving of hospital funds.

\section{G433(P) A CHILD WITH JUVENILE MYASTHENIA GRAVIS IN ASSOCIATION WITH TYPE 1 DIABETES MELLITUS}

E Woods, L Joseph, O Adeleye. Department of Paediatrics, Doncaster and Bassetlaw Hospitals NHS Trust, Worksop, UK

\subsection{6/archdischild-2020-rcpch.374}

Background and Aims Having an existing autoimmune disease predisposes to another; the underlying pathophysiology of this is unclear. Type 1 diabetes mellitus (T1DM) is an autoimmune condition which can be seen in conjunction with autoimmune thyroid disease, coeliac disease, autoimmune gastritis, vitiligo and Addison's disease. Myasthenia Gravis $(\mathrm{MG})$ is one autoimmune disorder where there are very few reported cases worldwide of its coexistence with T1DM. In this case, we report a child with T1DM who later develops MG.

Case Presentation Six months after initial diagnosis of T1DM, this 7 year old child presented with ocular symptoms, dysarthria and dysphagia during acute varicella zoster infection. Investigations were all negative including AChR, anti-musk and anti-ganglioside antibodies. Despite this, a trial of pyridostigmine provided symptomatic improvement. Clinical diagnosis of juvenile myasthenia was verified when a weaning dose of pyridostigmine resulted in re-emergence of clinical symptoms.

Outcome and Follow Up This child remains on an increased dose of pyridostigmine for symptom control. Clinical diagnosis of two distinct co-existent autoimmune diseases (T1DM and MG) has now been confirmed.

Discussion This child had increased likelihood of developing other autoimmune conditions with a diagnosis of T1DM, increased further by a strong family history of rheumatoid arthritis. Thyroid endocrinopathy and coeliac disease are more commonly associated with T1DM and, as a result, are tested for at diagnosis of T1DM. Screening tests for other autoimmune conditions are not routine, however clinical vigilance in identifying other autoimmune conditions is required.

Conclusion Children with T1DM are predisposed to other autoimmune conditions including, although extremely rarely, MG. In a child with existing autoimmune disease presenting with neurological symptoms suggestive of $\mathrm{MG}$, it is important to consider tests for juvenile MG, although lack of positive results does not rule out disease. The diagnosis of $\mathrm{MG}$ in children is largely clinical and diagnosis is usually based on response to pyridostigmine. 